DE

M E D I C I N A

T R O P I C A L

$\mathrm{DE}$

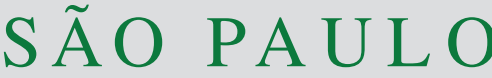

JOURNAL OF THE SÃO PAULO INSTITUTE OF TROPICAL MEDICINE

'Zhujiang Hospital of Southern Medical University, Department of Infectious Diseases, Guangzhou, Guangdong, China

Correspondence to: Xiaozhu Zhong Zhujiang Hospital of Southern Medical University, Department of Infectious Diseases, № 253, Industrial Avenue, Haizhu District, 510280, Guangzhou, Guangdong, China

E-mail: zjyyzhongxiaozhu@163.com

Received: 14 November 2019

Accepted: 8 January 2020

\section{Efficacy of antiviral therapy during the second or the third trimester for preventing mother-to-child hepatitis B virus transmission: a systematic review and meta-analysis}

\author{
Xiuhan Yang ${ }^{\circledR 1}$, Xiaozhu Zhong ${ }^{\circledR 1}$, Huihua Liao ${ }^{\circledR}$, Yongchang Lai ${ }^{\circledR 1}$
}

\section{ABSTRACT}

For pregnant women with high viral load, antiviral therapy has been administered in addition to active and passive immune prophylaxis as a crucial adjunctive therapy to interrupt mother-to-child hepatitis B virus (HBV) transmission (MTCT). However, the time of antiviral therapy onset remains controversial. A systematic review and meta-analysis was conducted to compare the efficacy of antiviral therapy during the second or the third trimester for prevention of HBV vertical transmission. We searched nine databases for observational studies and randomized controlled trials that enrolled pregnant women with positive HBsAg treated with antivirals. The outcomes of interest were maternal HBV-DNA levels prior to delivery and the rates of HBV MTCT. We included nine studies that enrolled 1,502 pregnant women. The average HBV-DNA level before treatment was approximately $8 \log _{10}$ copies/ $\mathrm{mL}$. Compared to the onset of antiviral intervention in the third trimester, the beginning of treatment in the second trimester distinctly reduced maternal predelivery HBV-DNA levels. However, no significant difference in HBV MTCT was found between the second and third trimester groups. Furthermore, the subgroup analysis showed that there were no significant differences between groups beginning treatment at different times (second or third trimester) with regard to HBV MTCT or other evaluated endpoints. For pregnant women with HBVDNA levels less than or equal to $8 \log _{10}$ copies/mL, the beginning of antiviral treatment can be delayed until the third trimester.

KEYWORDS: Antiviral therapy. Hepatitis B. Hepatitis B virus. Mother-to-child transmission.

\section{INTRODUCTION}

Chronic hepatitis B (CHB) remains a serious global public health issue ${ }^{1-5}$. According to data from the World Health Organization (WHO), an estimated 257 million people are infected with hepatitis B virus (HBV) ${ }^{3}$. Mother-to-child transmission (MTCT) is the major cause of CHB infection ${ }^{3,6,7}$. Prevention of HBV MTCT could therefore reduce the number of new HBV carriers. In addition, if HBV is left untreated, the risk of chronicity will depend on the age at which the patient acquired the infection $(80-90 \% \text { in newborns and }<5 \% \text { in adults })^{8}$. Compared to the acute hepatitis B infection, CHB is more likely to result in severe long-term life-threatening complications, including cirrhosis and hepatocellular carcinoma. In 2015, 887,000 deaths were reported and attributed to HBV complications ${ }^{9}$. Therefore, preventing MTCT could effectively decrease deaths related to CHB.

Recently, infants have been subjected to active and passive immune prophylactic measures, called timely HepB-BD, and the WHO recommends completion of the HBV vaccine schedule as a mainstream procedure to prevent $\mathrm{HBV}$ vertical transmission ${ }^{6}$. 
However, $5-10 \%$ of newborns are still infected by their HBV-positive mothers ${ }^{4,10}$. Infants born to mothers with high viral load (more than $10^{6}$ copies $/ \mathrm{mL}$ ) and hepatitis B envelope antigen ( $\mathrm{HBeAg}$ ) positivity showed 10-30\% immune prophylaxis failure, while those born to mothers with low maternal viral load (less than $10^{6}$ copies $/ \mathrm{mL}$ ) show less than $3 \%$ failure ${ }^{8,10}$. Thus, antiviral intervention for pregnant women with high maternal HBV-DNA levels will help to achieve a global eradication of CHB infection ${ }^{10-12}$.

Although a consensus on the use of antiviral drugs to interrupt HBV MTCT has been reached, the proper timing of antiviral treatment during pregnancy remains controversial. Antiviral therapy is typically administered in the second and third trimesters of gestation. According to the American Association for the Study of Liver Diseases (AASLD) ${ }^{13}$ and the Asian Pacific Association for the Study of the Liver (APASL) ${ }^{14}$, the starting point for maternal treatment should be at 28-32 weeks of gestation. The European Association for the Study of the Liver (EASL) ${ }^{15}$ and the Chinese Medical Association ${ }^{15,16}$ recommend treatment initiation at 24-28 weeks of gestation. However, related cohort studies with large sample sizes and randomized controlled trials (RCTs) are still lacking. Therefore, a systematic review and meta-analysis was conducted to compare the efficacy of antiviral therapy for HBV MTCT prevention in the second or in the third trimester.

\section{METHODS}

\section{Search strategy}

A comprehensive search of PubMed, Embase, the Cochrane Library, Ovid MEDLINE, Web of Science, SinoMed, the China National Knowledge Infrastructure (CNKI), the China Science and Technology Journal Database and the Wanfang Database was conducted from January 2013 to December 2018. The following search terms and their variations were used: "pregnant or mother", "hepatitis B virus or HBV or chronic hepatitis B or CHB", "antiviral or lamivudine or LAM or telbivudine or LdT or tenofovir or TDF", "mother-to-child or MTCT or vertical or mother-to-fetus or maternal-neonatal" and "infection or transmission or prevention or control". No language restrictions were applied. The reference lists were reviewed to identify additional articles.

\section{Eligibility criteria}

Studies were included if they met the following inclusion criteria: 1) they were RCTs or cohort studies; 2) they included pregnant women with HBV infections who had accepted antiviral therapy during pregnancy; 3) they included only infants who received one dose of HBIG at birth and three doses of hepatitis B vaccine at birth or after delivery and 4) they included data on the MTCT rate.

MTCT was considered to have occurred if HBsAg positivity was observed or if HBV-DNA levels were detectable when the infants were seven to 12 months old.

The following types of studies were excluded: 1) case reports, review articles, letters to the editor, editorials and conference abstracts; 2) studies in which patients were coinfected with HIV, HCV or HDV, or patients were not pregnant women; 3 ) studies in which patients received a combined-HBIG injection during pregnancy or 4) studies in which antiviral therapy was initiated before pregnancy or during early pregnancy (before the second trimester).

\section{Data extraction}

Two independent investigators examined the titles and abstracts. The full-text versions of the included articles were then reviewed, following the same procedure. Disagreements were reconciled by a third person. The following information was extracted from each study: authorship, publication year, the age of subjects (years), maternal HBV-DNA levels before antiviral intervention and prior to delivery, baseline ALT level, intervention and control subject numbers, the time of treatment onset (in gestational weeks), the time of treatment discontinuation (in postpartum weeks), the study design and MTCT rates. In the included trials, the second trimester referred to 13-27 weeks of gestation, while the third trimester referred to the period beginning at 28 weeks of gestation. The specific weeks of gestation during which antiviral therapy was initiated and the average duration of antiviral treatment were obtained directly from the articles. The outcomes were the maternal HBV-DNA levels at delivery and the MTCT rate. The different groups of maternal HBV-DNA level were further divided into subgroups according to their specific antiviral initiation time. MTCT was considered to have occurred if HBsAg positivity was observed or if HBV-DNA levels were detectable when the infants were seven to 12 months old. Then, two analyses were carried out according to subgroups: the analysis on the differences in efficacy among various antiviral initiation times and the analysis on the differences in MTCT rates when HBsAg with respect to HBV-DNA seropositivity was used as the outcome.

\section{Risk of bias assessment}

The Newcastle-Ottawa Scale (NOS) was used to assess the quality of the included observational studies. NOS 
involves a total of eight items divided into three categories: selection (4), comparability (1) and exposure (3). The scale ranges from zero to nine points. The studies that met the requirements were awarded the corresponding numbers of points. A maximum of two stars were given for the comparability parameter. A study with a score of more than 5 points was considered of high quality.

\section{Statistical analysis}

We performed the review and meta-analysis according to the recommendations of the Cochrane Collaboration. To create forest plots, we calculated the risk ratio (RR) and $95 \%$ confidence interval $(95 \% \mathrm{CI})$ using a binomial distribution for dichotomized outcomes, and we determined the weighted mean difference (WMD) with $95 \%$ CI for continuous outcomes. Before we analyzed the data, we tested the heterogeneity among the included studies by means of the chi-square test and $I^{2}$ statistic. $P<0.05$ for the chi-square test or an $I^{2}>50 \%$ were considered as indicative of high heterogeneity. If high heterogeneity was observed, a random effect model (DerSimonian-Laird method) was used to analyze the data. In the absence of significant heterogeneity, a fixed model (Mantel-Haenszel method) was applied. When considerable heterogeneity was found, a sensitivity analysis was performed by omitting one study in each iteration to explore the influence of a single study on the overall estimate. A $P$ value less than 0.05 was judged as statistically significant. All statistical analyses were conducted using the Review Manager software, version 5.3 (Cochrane Collaboration, Copenhagen, Denmark.

\section{RESULTS}

The search strategy identified 6,232 papers. After 1,777 duplicate articles were removed, 4,455 articles remained, 4,414 of which were excluded on the basis of the title and abstract. Then, the full texts of the remaining 41 studies were examined. Thirty-two studies were removed: 12 were related to drug withdrawal; 11 were review articles, conference abstracts or reports; seven involved different interventions and two reported different outcomes of interest. Ultimately, nine studies that met the inclusion criteria were included in this meta-analysis.

\section{Characteristics of the included studies}

The details of the study selection process are described in Figure 1. All the included articles were observational cohort studies. The majority of these studies were reported

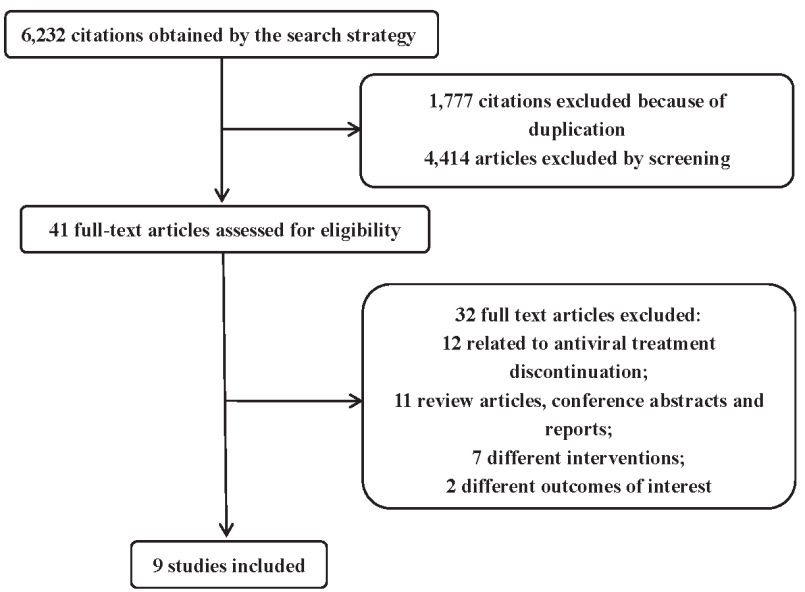

Figure 1 - Flow diagram of the study selection process.

in Chinese $(\mathrm{n}=5)^{17-21}$, while four ${ }^{22-25}$ were reported in English. Overall, nine studies that enrolled a total of 1,502 mothers were included. Most of these studies used telbivudine (LdT) and tenofovir disoproxil fumarate (TDF). All of the infants were born in China and received active and passive combined immunization with 100 IU HBIG once in the neonatal period and hepatitis B vaccine three times, at zero, one and six months postpartum.

Predelivery maternal HBV-DNA levels ( $\log _{10}$ scale) after treatment initiation in the second trimester compared with the third trimester

All the articles ${ }^{17-25}$ provided detailed descriptions on the maternal HBV-DNA levels before intervention (Table 1). There were no statistically significant differences in maternal HBV-DNA levels $\left(\log _{10}\right.$ scale) before treatment between mothers who began the antiviral therapy in the second trimester and those who began it in the third trimester $\left(I^{2}=0 \%, P=0.25\right.$, Table 2$)$. Only six studies ${ }^{18-21,23,25}$ reported maternal HBV-DNA levels prior to delivery. The pre-delivery maternal HBV-DNA levels ( $\log _{10}$ scale) differed significantly $(P<0.001)$ between the two groups, with distinguished heterogeneity $\left(P<0.001, I^{2}=82 \%\right.$, Table 3). Therefore, we performed the subgroups analysis according to the different drug treatment initiation times (Table 4), which reduced the heterogeneity $\left(I^{2}=0 \%\right.$ for each subgroup), and evaluated the stability of the results. The results indicated that beginning the antiviral therapy in the second trimester significantly reduced the HBV-DNA levels in HBV-infected mothers $(P<0.01$ for most subgroup, $W M D=-0.93,95 \% C I-1.08-0.77)$.

Thus, compared to the beginning of treatment in the third trimester, the onset of antiviral intervention in the second trimester distinctly reduced maternal HBV-DNA levels, and this effect was related to the antiviral treatment. 


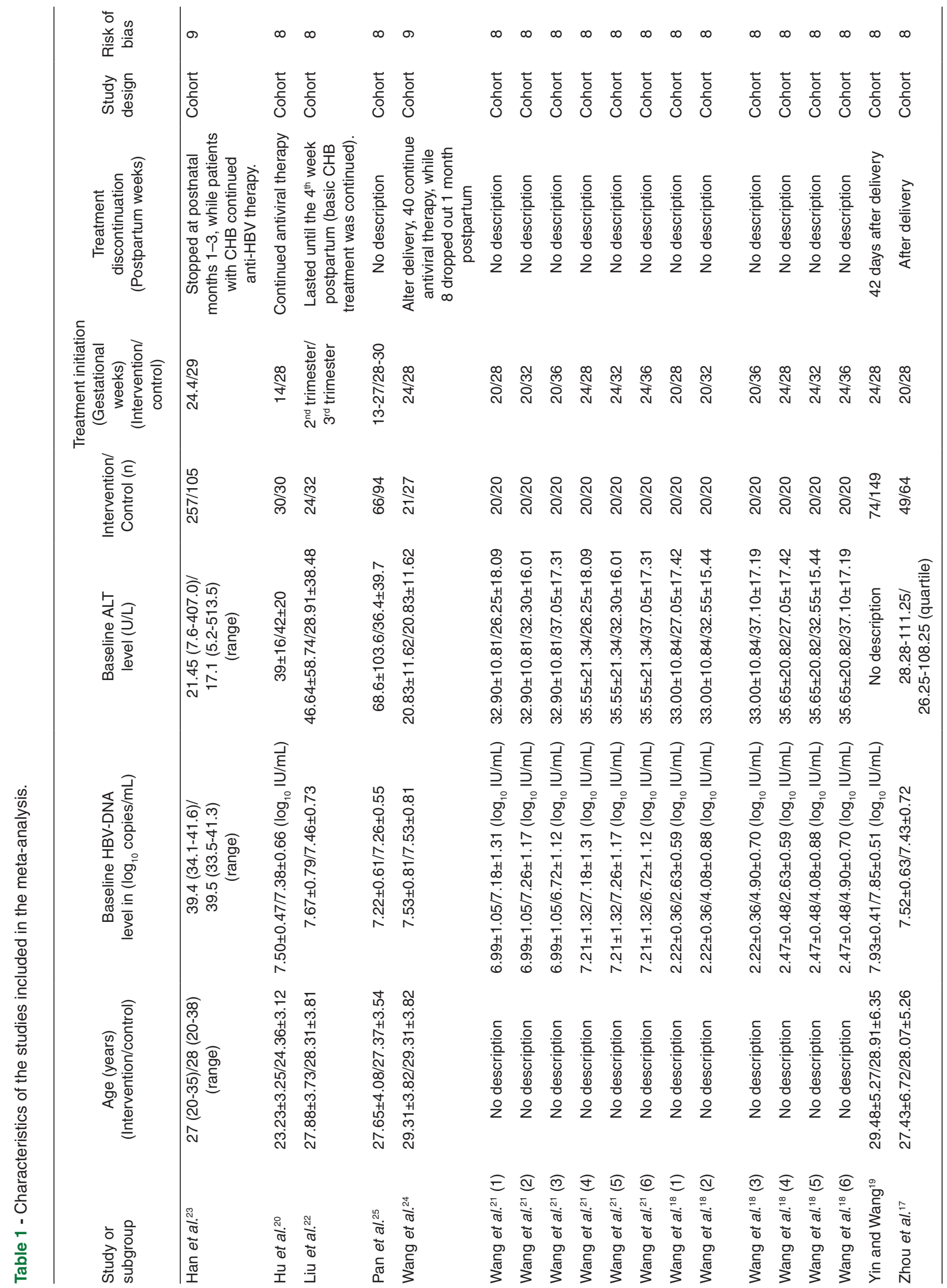


Table 2 - Antiviral intervention initiated in the second trimester compared to the third trimester in maternal HBV-DNA (log ${ }_{10}$ ) before treatment.

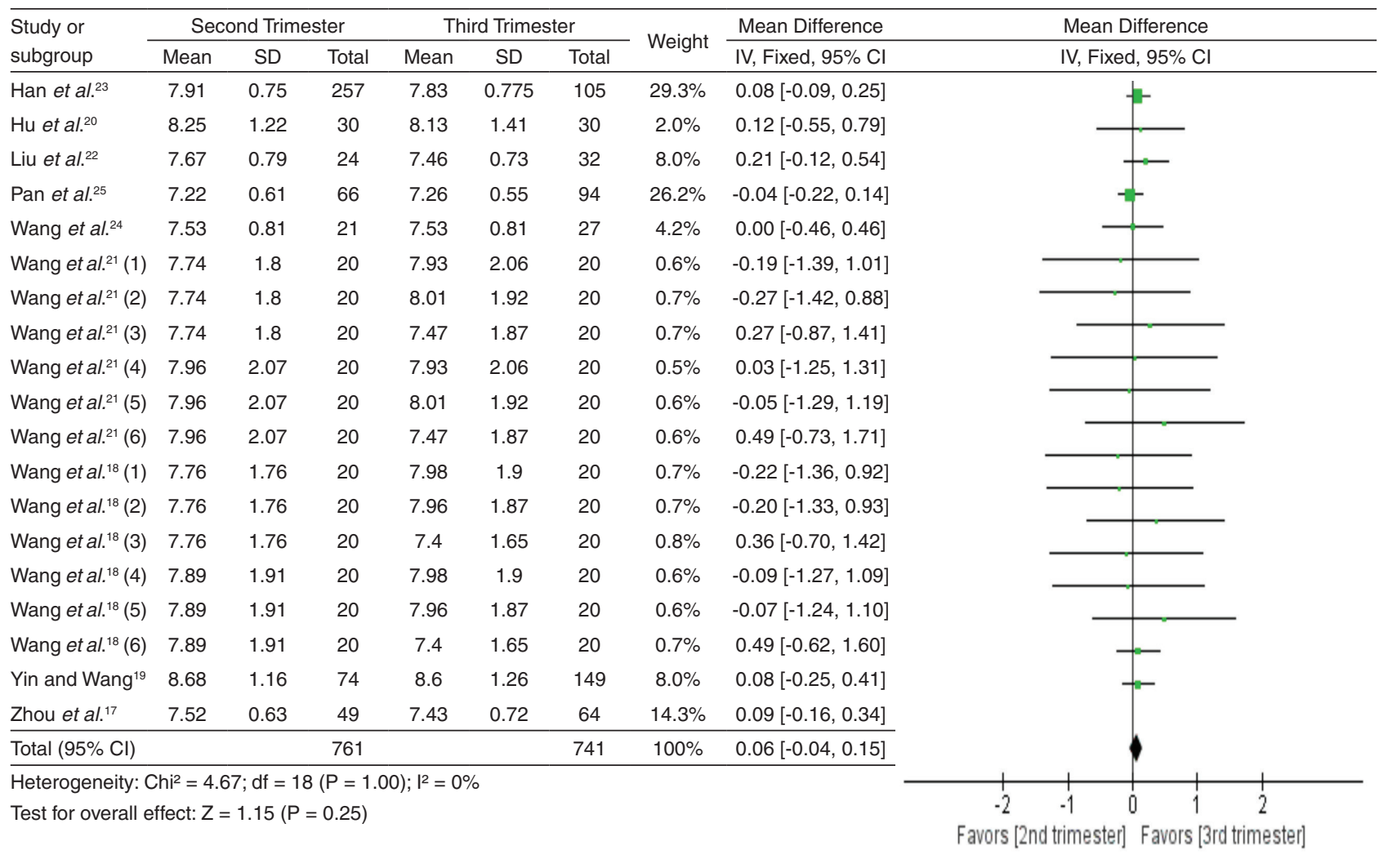

Table 3 - Antiviral intervention initiated in the second trimester compared to the third trimester in maternal HBV-DNA (log ${ }_{10}$ ) prior to delivery.

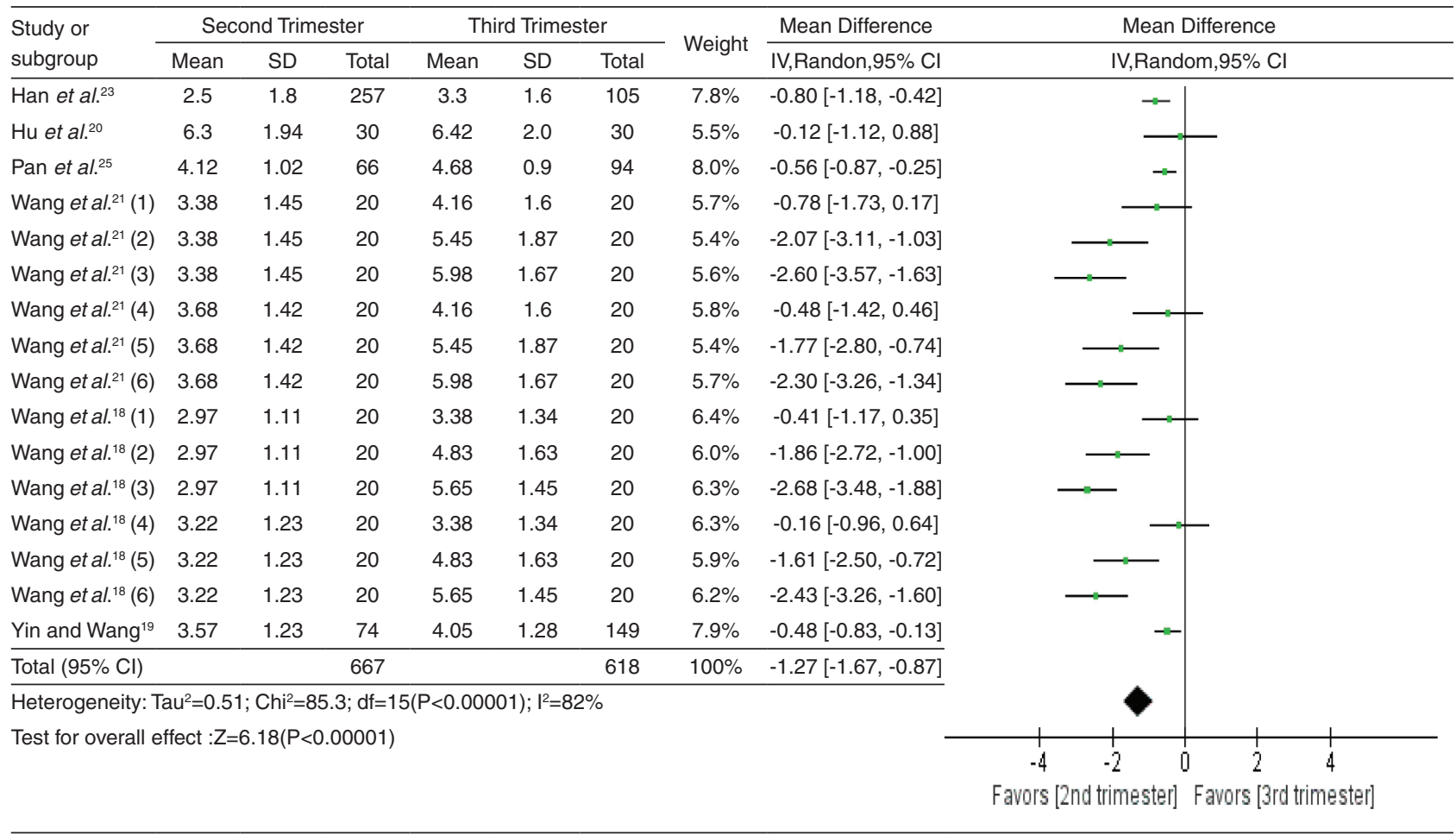


Table 4 - Forest plot of antiviral intervention initiated in the second trimester compared to the third trimester with respect to maternal HBV-DNA ( $\log _{10}$ ) prior to delivery (subgroups analyses according to different starting times).

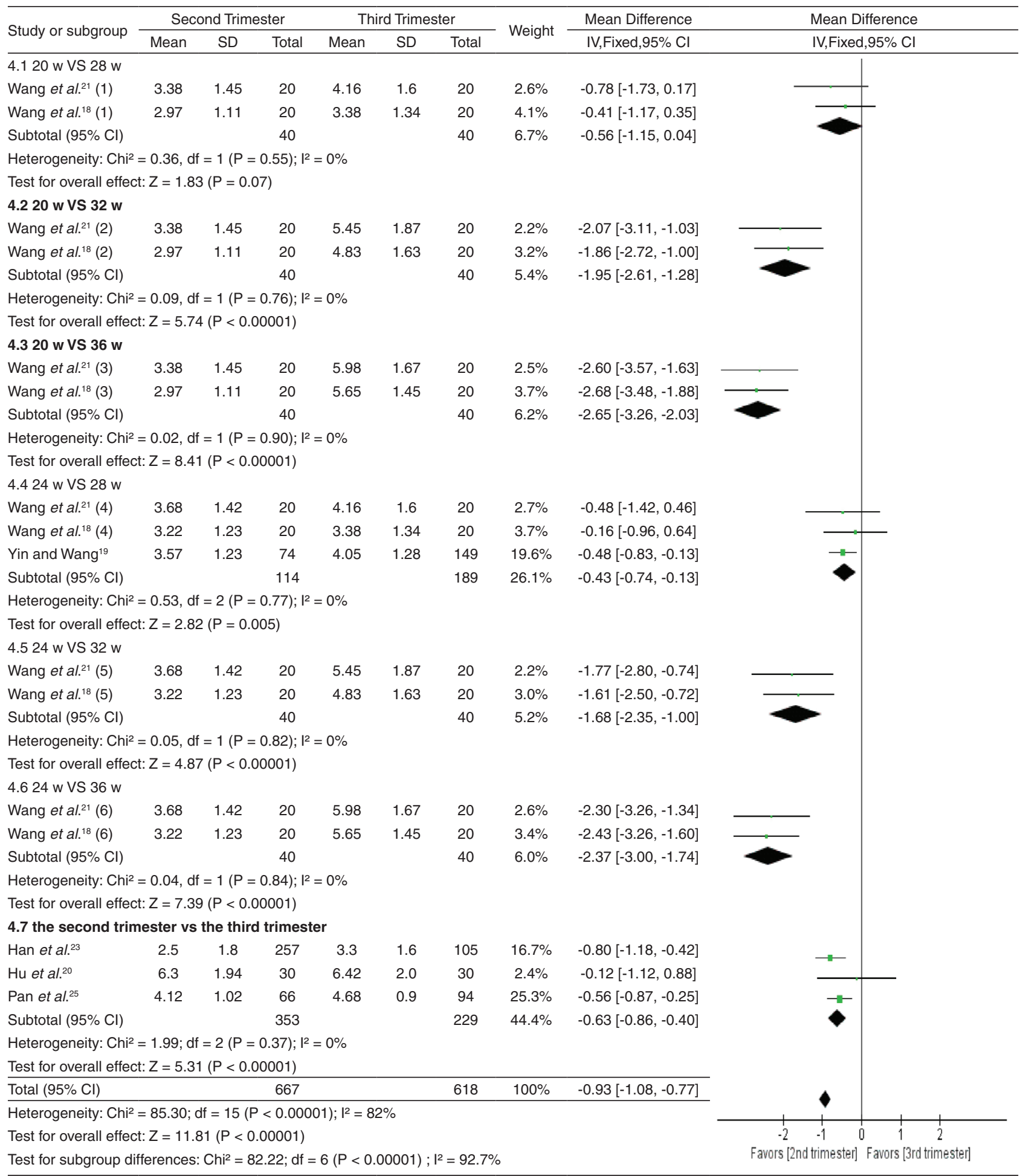

Relative risk of mother-to-child transmission with antiviral therapy initiation in the second vs. third trimester

Among the 1,502 enrolled pregnant women, 761 began to receive antiviral treatment in the second trimester, while 741 received it in the third trimester. The percentages of newborns with HBsAg or HBV-DNA seropositivity in the second trimester group and third trimester group were $0 \%$ 
Table 5 - Forest plot of HBV MTCT rate (the second trimester or the third trimester).

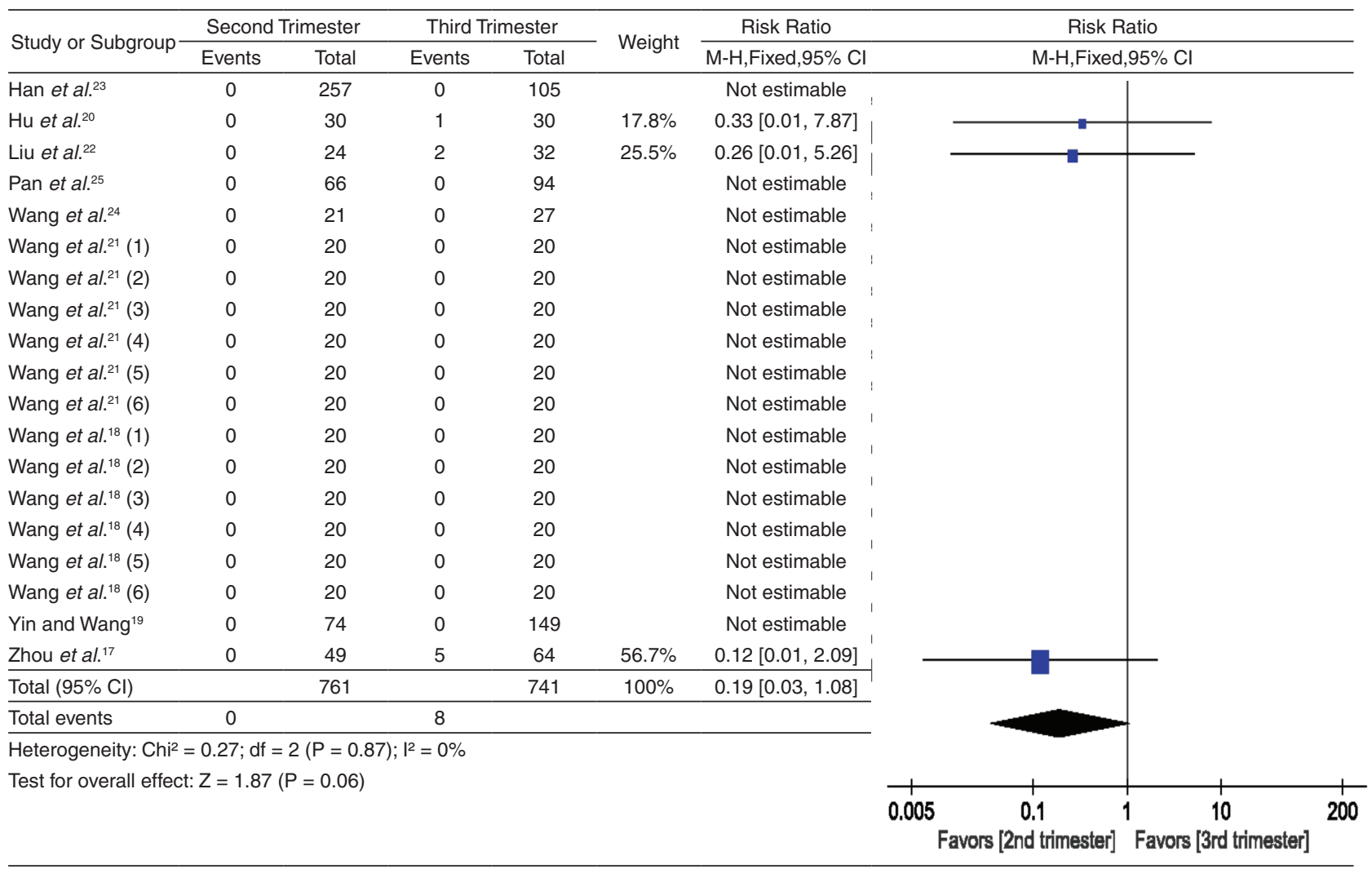

$(0 / 761)$ and $1.08 \%(8 / 741)$, respectively. The rate of HBV MTCT in the second trimester group was similar to the one in the third trimester group, with no significant difference between the groups $\left(R R=0.19,95 \%\right.$ CI $0.03-1.08, I^{2}=0 \%$, $P=0.06$, Table 5).

To compare the effects of different antiviral drugs initiation times on HBV MTCT rates, we divided the data into eight subgroups according to the time at which the intervention was initiated (Table 6). The results of the different subgroups suggested that different durations of antiviral treatment led to similar incidences of HBV MTCT $(P>0.05)$.

To assess whether HBsAg seropositivity and HBV-DNA seropositivity were equivalent endpoints for the evaluation of HBV MTCT, we split the data into two subgroups. Among the nine included articles, eight papers ${ }^{17-19,21-25}$ reported the HBsAg seropositivity of the infants, while seven papers ${ }^{17,18,21-25}$ reported the HBV-DNA seropositivity in the infants. In the HBsAg group, the HBV MTCT rates in the second trimester and the third trimester were $0 \%(0 / 731)$ and $0.98 \%$ (7/711), respectively. The rate of HBV MTCT in the second trimester was not significantly lower than the one in the third trimester $\left(R R=0.16,95 \% C I 0.02-1.28, I^{2}=0 \%\right.$, $P=0.08$ ) (Table 7). In the HBV-DNA seropositivity group, the HBV MTCT rates in the second and third trimesters were $0 \%(0 / 657)$ and $0.53 \%$ (3/562), respectively (Table 7), with no significant difference between the trimesters ( $R R=0.19,95 \% C I 0.01-3.51, P=0.26)$. Thus, with regard to HBV MTCT prevention, there is no significant difference in MTCT rates when HBsAg with respect to HBV-DNA seropositivity was used as the outcome.

\section{DISCUSSION}

This meta-analysis had two major findings. Firstly, compared to the treatment initiated in the third trimester, the antiviral intervention initiated in the second trimester could distinctly reduce maternal HBV-DNA levels, and this effect was closely related to the time of initiation. In addition, second-trimester and third-trimester initiations of antiviral treatment yielded similar incidences of HBV MTCT, irrespective of the initiation time and the different studied outcomes.

Lamivudine, LdT and TDF are equivalent in terms of antiviral efficacy ${ }^{6,26}$. Hence, in our study, their effects were merged and directly compared. In addition, intrauterine infection can be almost perfectly prevented when the HBV-DNA load of the mother is $<10^{4}$ copies $/ \mathrm{mL}$ 
Table 6 - Forest plot on the rate of mother-to-child hepatitis B virus transmission (subgroup analyses according to different starting times)

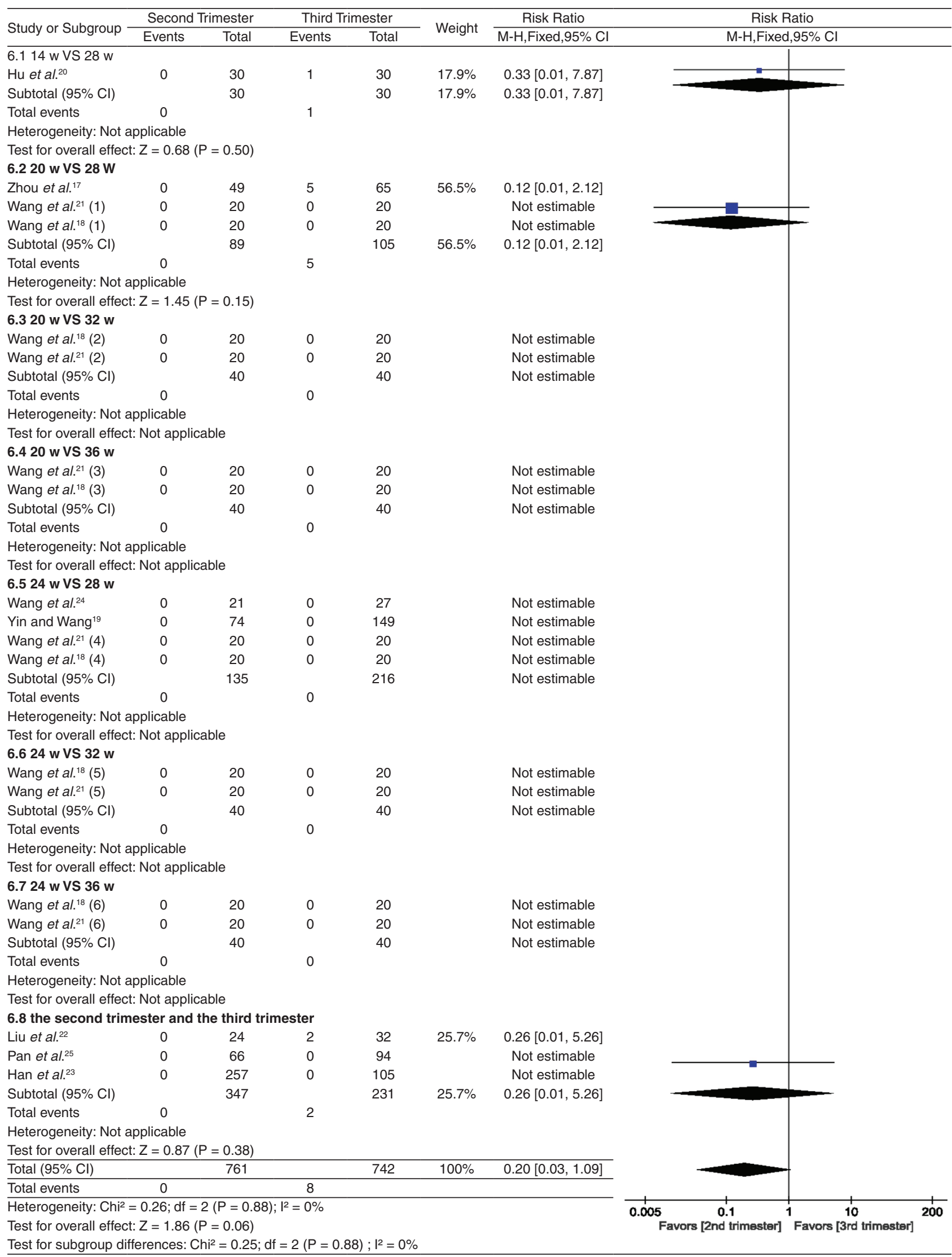


Table 7 - Forest plot on the rate of mother-to-child hepatitis B virus transmission (HBsAg seropositivity or HBV-DNA seropositivity).

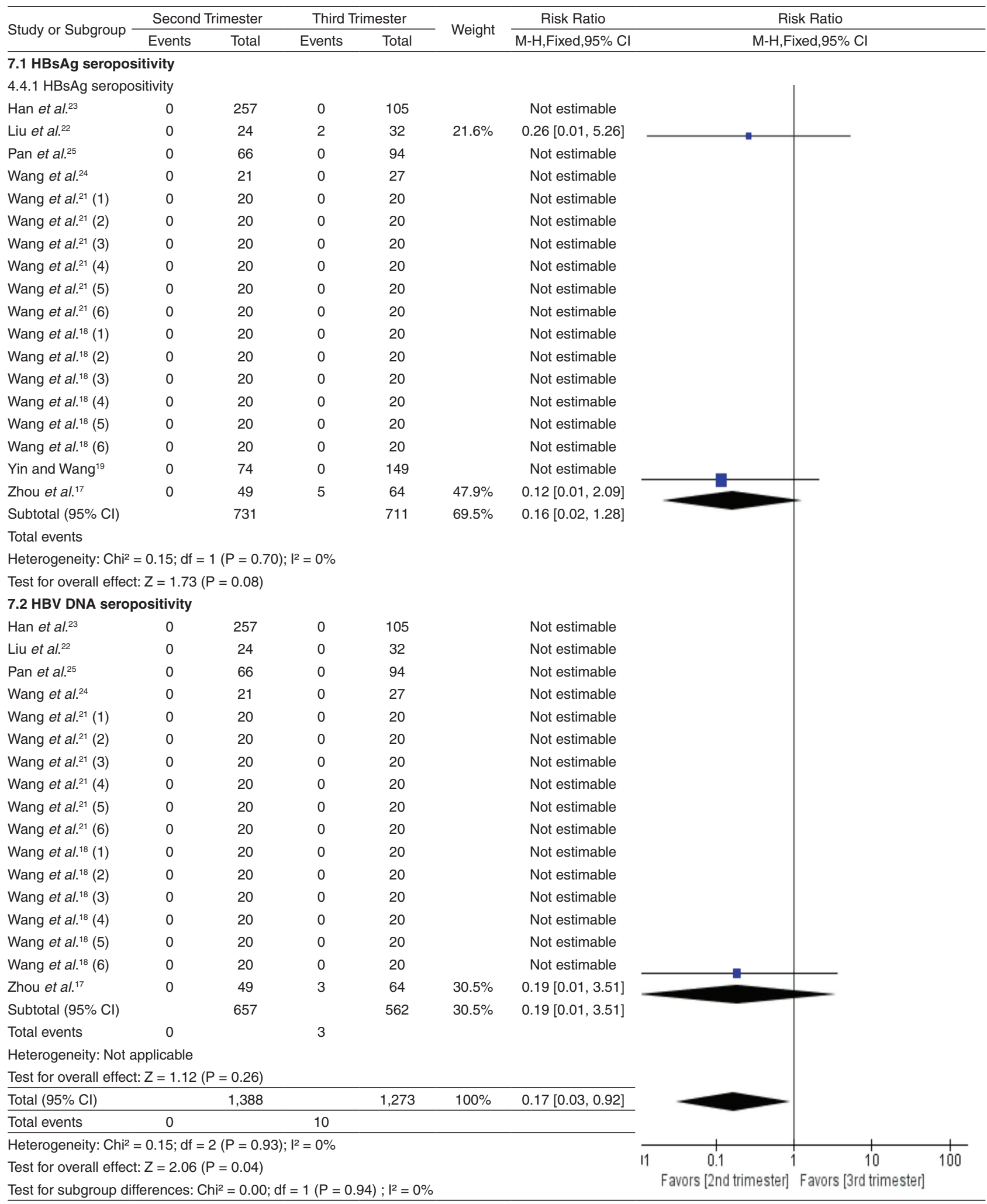

$\left(10^{3} \mathrm{IU} / \mathrm{mL}\right)^{27-29}$, which is considered a safety maternal pre-delivery HBV-DNA threshold for HBV MTCT prevention. Among the included studies, the maternal pre- delivery HBV-DNA levels in mothers that began antiviral therapy in the second trimester were approximately $3 \log _{10}$ copies/mL $18,19,21$, while the corresponding 
values in the third trimester were approximately $4 \log _{10}$ copies/mL $18,19,21,25$. Both groups were within the safety threshold. This finding could explain why the antiviral therapy initiated in the second trimester, in contrast with the one initiated in the third trimester, could significantly reduce the maternal pre-delivery HBV-DNA levels without changing MTCT.

However, some results should not be ignored. The $P$ value for the difference in HBV MTCT rate between the second and the third trimester group was 0.06 , which was close to the threshold for a statistically significant difference (0.05). Furthermore, in the included studies, the average maternal HBV- DNA level before treatment was approximately $8 \log _{10}$ copies $/ \mathrm{mL}$. In mothers who began the antiviral intervention in the third trimester, the average maternal HBV- DNA level prior to delivery was approximately $4 \log _{10}$ copies $/ \mathrm{mL}$, close to the safety threshold for HBV MTCT prevention. These findings suggest that the initiation of antiviral treatment can be delayed until the third trimester in mothers in whom HBVDNA levels are less than or equal to $8 \log _{10}$ copies $/ \mathrm{mL}$. This type of treatment with a shorter duration, fewer side effects and lower financial burden, will be more suitable than second trimester-initiated treatment for the widespread use to prevent HBV MTCT. However, if the HBV-DNA level in a mother exceeds $8 \log _{10}$ copies $/ \mathrm{mL}$ and antiviral therapy is initiated in the third trimester, the risk of HBV MTCT may increase. Larger sample size cohort studies, including subjects with higher maternal HBV-DNA levels are needed to verify this hypothesis.

Recently, two similar meta-analyses compared the efficacy of antiviral treatment started in the second trimester with the one strated on the third trimester for preventing HBV MTCT. In 2014, a study ${ }^{30}$ reported that antiviral intervention is effective in preventing HBV MTCT whether it is started in the second or the third trimester, with no significant difference in the rate of vertical transmission. In 2018, another study ${ }^{6}$ reported that antiviral intervention initiated in the second trimester might be better than the one initiated in the third trimester, as indicated by the HBVDNA seropositivity rather than the HBsAg seropositivity in infants. In the latter study, the time to diagnosis of MTCT was within $24 \mathrm{~h}$ after delivery, which may have led to an overestimation of the MTCT rate ${ }^{30-32}$. Therefore, in our study, MTCT was considered to have occurred if HBsAg positivity was observed or if HBV- DNA levels were detectable when infants were seven to 12 months old.

This meta-analysis has four advantages. Above all, we searched for comprehensive databases, updated the included articles and merged the effects of different antiviral drugs. Moreover, all the included studies were parallel control trials, which have greater power than single-arm studies. Most importantly, this study is the first to interpret why initiating antiviral therapy in the second trimester rather than in the third trimester can distinctly reduce maternal pre-delivery HBV-DNA levels without changing MTCT. Finally, we divided maternal HBV-DNA levels prior to delivery and the HBV MTCT incidences into several subgroups according to specific drug intervention times to further explore associations of specific treatment durations with the examined outcomes.

The present study also has some limitations. Firstly, there is a lack of high-quality RCTs and cohort studies with larger sample sizes because of ethical restrictions, among other reasons. Secondly, different countries have different vaccination methods and MTCT diagnostic criteria. To improve the comparability, our inclusion criteria limited studies carried out with different methods. Thus, even though there was no language restriction, all the study participants were Asian. These limitations need to be considered when evaluating the conclusions.

\section{CONCLUSION}

For pregnant women with HBV-DNA levels less than or equal to $8 \log _{10}$ copies $/ \mathrm{mL}$ initiation of antiviral treatment can be delayed until the third trimester of pregnancy.

\section{REFERENCES}

1. Pan CQ, Duan Z, Dai E, Zhang S, Han G, Wang Y, et al. Tenofovir to prevent hepatitis $\mathrm{B}$ transmission in mothers with high viral load. New Engl J Med. 2016;374:2324-34.

2. Hu Y, Xu C, Xu B, Hu L, Liu Q, Chen J, et al. Safety and efficacy of telbivudine in late pregnancy to prevent mother-to-child transmission of hepatitis B virus: a multicenter prospective cohort study. J Viral Hepat. 2018;25:429-37.

3. Chen HL, Zha ML, Cai JY, Qin G. Maternal viral load and hepatitis $B$ virus mother-to-child transmission risk: a systematic review and meta-analysis. Hepatol Res. 2018;48:788-801.

4. Samadi Kochaksaraei G, Castillo E, Osman M, Simmonds K, Scott AN, Oshiomogho JI, et al. Clinical course of 161 untreated and tenofovir-treated chronic hepatitis B pregnant patients in a low hepatitis B virus endemic region. J Viral Hepat. 2016;23:15-22.

5. Sun W, Zhao S, Ma L, Hao A, Zhao B, Zhou L, et al. Telbivudine treatment started in early and middle pregnancy completely blocks HBV vertical transmission. BMC Gastroenterol. 2017; 17:51.

6. Song J, Yang F, Wang S, Tikande S, Deng Y, Tang W, et al. Efficacy and safety of antiviral treatment on blocking the mother-to-child transmission of hepatitis B virus: a meta-analysis. J Viral Hepat. 2018;26:397-406. 
7. Brown RS Jr, McMahon BJ, Lok AS, Wong JB, Ahmed AT, Mouchli MA, et al. Antiviral therapy in chronic hepatitis B viral infection during pregnancy: a systematic review and meta-analysis. Hepatology. 2016;63:319-33.

8. Hyun MH, Lee YS, Kim JH, Je JH, Yoo YJ, Yeon JE, et al. Systematic review with meta-analysis: the efficacy and safety of tenofovir to prevent mother-to-child transmission of hepatitis B virus. Aliment Pharmacol Ther. 2017;45:1493-505.

9. Yi W, Li MH, Xie Y, Wu J, Hu YH, Zhang D, et al. Prospective cohort study on the efficacy and safety of telbivudine used throughout pregnancy in blocking mother-to-child transmission of hepatitis B virus. J Viral Hepat. 2017;24 Suppl 1:49-56.

10. Ayres A, Yuen L, Jackson KM, Manoharan S, Glass A, Maley $\mathrm{M}$, et al. Short duration of lamivudine for the prevention of hepatitis B virus transmission in pregnancy: lack of potency and selection of resistance mutations. J Viral Hepat. 2013;21:80917.

11. Han GR, Jiang HX, Wang CM, Ding Y, Wang GJ, Yue X, et al. Long-term safety and efficacy of telbivudine in infants born to mothers treated during the second or third trimesters of pregnancy. J Viral Hepat. 2017;24:514-21.

12. Chen HL, Lee CN, Chang CH, Ni YH, Shyu MK, Chen SM, et al. Efficacy of maternal tenofovir disoproxil fumarate in interrupting mother-to-infant transmission of hepatitis B virus. Hepatology. 2015;62:375-86.

13. Terrault NA, Lok AS, McMahon BJ, Chang KM, Hwang JP, Jonas $\mathrm{MM}$, et al. Update on prevention, diagnosis, and treatment of chronic hepatitis B: AASLD 2018 hepatitis B guidance. Hepatology. 2018;67:1560-99.

14. Sarin SK, Kumar M, Lau GK, Abbas Z, Chan HL, Chen CJ, et al. Asian-Pacific clinical practice guidelines on the management of hepatitis B: a 2015 update. Hepatol Int. 2016;10:1-98.

15. Idilman R. Management of special patient groups with hepatitis $B$ virus infection: The EASL 2017 Clinical Practice Guidelines. Turk J Gastroenterol. 2017;28:518-21.

16. Hou J, Cui F, Ding Y, Dou X, Duan Z, Han G, et al. Management algorithm for interrupting mother-to-child transmission of hepatitis B virus. Clin Gastroenterol Hepatol. 2019;17:192936.

17. Zhou DS, Lin QX, Jiang JX. Effect of Lamivudine blocking mother-to-child transmission of HBV in different pregnancy stages. Hainan Med J. 2013;24:3155-7.

18. Wang H, Li H, Yang X, Gong M, Gao S, Zhang F, Xia J, et al. Efficacy and safety on blocking HBV vertical transmission by oral tenofovir disoproxil treatment in middle-late pregnancy. Chin J Exp Clin Infect Dis. 2018;12:51-5.
19. Yin Y, Wang M. Efficacy of nucleos(t)ide analogue antiviral therapy on interrupting mother-to-child transmission of hepatitis B virus. Chin J Exp Clin Infect Dis. 2018;12:169-73.

20. Hu M, Zhuang L, Wang J, Li L, Yang Y, Bai B, et al. The efficacy and safety of tenofovir on blocking mother-infant transmission of hepatitis B. Chin J Drug Depend. 2018;27:379-83.

21. Wang HB, Li H, Yang XD, Gong M, Gao SY, Zhang FR, et al. Clinical observation of effectiveness and safety on blocking HBV vertical transmission via oral telbivudine treatment in middle-late pregnancy. J Pract Med. 2016;32:3928-31.

22. Liu Y, Wang M, Yao S, Yuan J, Lu J, Li H, et al. Efficacy and safety of telbivudine in different trimesters of pregnancy with high viremia for interrupting perinatal transmission of hepatitis B virus. Hepatol Res. 2016;46:E181-8.

23. Han GR, Jiang HX, Yue X, Ding Y, Wang CM, Wang GJ, et al. Efficacy and safety of telbivudine treatment: an open-label, prospective study in pregnant women for the prevention of perinatal transmission of hepatitis B virus infection. J Viral Hepat. 2015;22:754-62.

24. Wang J, Liu J, Qi C, Yan T, Cao F, Jin L, et al. Efficacy of tenofovir disoproxil fumarate to prevent vertical transmission in mothers with lamivudine-resistant HBV. Antivir Ther. 2015;20:681-7.

25. Pan CQ, Yi W, Liu M, Wan G, Hu YH, Zhou MF. Lamivudine therapy during the second vs the third trimester for preventing transmission of chronic hepatitis B. J Viral Hepat. 2017;24:24652.

26. Greenup AJ, Tan PK, Nguyen V, Glass A, Davison S, Chatterjee $\mathrm{U}$, et al. Efficacy and safety of tenofovir disoproxil fumarate in pregnancy to prevent perinatal transmission of hepatitis B virus. J Hepatol. 2014;61:502-7.

27. Xu H, Zeng T, Liu JY, Lei Y, Zhong S, Sheng YJ, et al. Measures to reduce mother-to-child transmission of hepatitis $\mathrm{B}$ virus in China: a meta-analysis. Dig Dis Sci. 2014;59:242-58.

28. Xiaoguang D. Strategy for anti-HBV therapy in women of childbearing age and pregnancy. Chin J Hepatol. 2015; 23:801-2.

29. Zhang Y, Guo PL, Wang QQ, Han ZY, Hou HY. Association between viral load and pregnancy outcome in women with chronic hepatitis B. J Sun Yat-Sen Univ Med Sci. 2014;35:866-9.

30. Lu YP, Lung XJ, Xiao XM, Huang SM, Liu ZW, Li J, et al. Telbivudine during the second and third trimester of pregnancy interrupts HBV intrauterine transmission: a systematic review and meta-analysis. Clin Lab. 2014;60:571-86.

31. But DY, Yuen MF, Fung J, Lai CL. Safety evaluation of telbivudine. Expert Opin Drug Saf. 2010;9:821-9.

32. Jonas MM. Hepatitis B and pregnancy: an underestimated issue. Liver Int. 2009;29 Suppl 1:133-9. 